\title{
Wagniskapital: Mit Erfahrung zum Erfolg
}

\section{Margaret Green}

\section{Relevanz}

Wagniskapital finanziert die innovativsten Unternehmen mit dem grössten Risiko und trimmt diese mit Beratung und Kontrolle auf Erfolg. So beschleunigt die Wagnisfinanzierung die Erneuerung der Wirtschaft. Damit die positiven Wirkungen zustande kommen, braucht es Reputation und einen Erfolgsausweis, damit sich die besten Start-ups anstellen und die Wagniskapitalgesellschaften sich leichter refinanzieren können. Was könnte mehr Reputation bringen als Erfolg? Auch die Wagnisfinanziers werden aus Erfahrung klug. Eine erfolgreiche Investition ist der beste Beweis für die eigene Leistungsfähigkeit. Gerade für junge Wagniskapitalfonds ist es wichtig, mit einer Investition zur richtigen Zeit am richtigen Ort einen ersten Erfolg zu landen, der das nachfolgende Geschäft erleichtert.

\section{Quelle}

Nanda, Ramana, Sampsa Samila und Olav Sorenson (2020), The Persistent Effect of Initial Success: Evidence from Venture Capital, Journal of Financial Economics, erscheint demnächst.

Risikokapitalgeber finanzieren Innovation und Wachstum in der Wirtschaft. Junge und innovative Startups sind mangels Erfahrung und wegen fehlender Sicherheiten für klassische Finanzierungsformen wie Bankkredite oftmals zu riskant. Sie sind

M. Green $(\bowtie)$

Universität St.Gallen, St.Gallen, Schweiz

E-Mail: margaret.green@student.unisg.ch 
daher auf Wagniskapitalgeber angewiesen, die Beteiligungskapital geben, Risiko übernehmen und ihre Unternehmen bei Innovation und Wachstum unterstützen. Je erfolgreicher ein Start-up ist, desto rentabler ist die Investition der Finanziers. Bei Erfolg steigt der Risikokapitalgeber durch Verkauf seiner Anteile an andere Investoren und im besten Fall durch einen Börsengang aus dem Unternehmen aus. Ihr Kapital und ihre Expertise wird bei den nächsten Startups benötigt. Der Börsengang ist den besten Jungunternehmen vorbehalten. Die Wagniskapitalgeber erzielen bei einem Börsengang in den USA durchschnittlich $400 \%$ Bruttorendite, bei Verkäufen an andere Investoren liegt diese noch bei rund $143 \%$. Bei Misserfolg müssen sie allerdings ihre Investition meist ganz abschreiben.

Macht Erfolg erfolgreich? Wenn ein Wagniskapitalgeber einmal einen grossen Erfolg landet, kann man auch bei der nächsten Investition mit Erfolg rechnen. Die langanhaltende Erfolgsbeständigkeit ist eine Besonderheit des Wagniskapitals. Woher kommt ein anhaltender Erfolg bei riskanten Unternehmensinvestitionen? Die Wissenschaftler Ramana Nanda, Sampsa Samila und Olav Sorenson von den Universitäten Harvard, Yale, und IESE (Barcelona) gehen dieser Frage nach. Sie untersuchen, inwiefern der erste Erfolg eines Risikokapitalgebers den Erfolg ihrer weiteren Investitionen vorprogrammiert.

Dazu analysieren die Forscher Investitionen von 895 US-amerikanischen Wagniskapitalgebern zwischen 1961 und 2008. Eine Investition gilt dann als erfolgreich, wenn es bis 2016 zu einem Börsengang oder einem Verkauf des Jungunternehmens kam. Da Wagnisfinanziers typischerweise mit mehreren Finanzierungsrunden in dasselbe Unternehmen investieren, beschränken sich die Autoren auf die Erstinvestition in ein Unternehmen. Der Datensatz umfasst mehr als $46^{\prime} 000$ Investitionen.

Rund $51 \%$ der Unternehmen, die durch Wagniskapital finanziert wurden, gingen an die Börse oder wurden an andere Investoren verkauft. Die Wahrscheinlichkeit eines Börsengangs betrug rund $20 \%$. Mit dem Ausstieg durch Verkauf ihrer Anteile können die Finanziers den Ertrag auf ihre riskante Investition realisieren.

Im Schnitt ist jedes zweite wagnisfinanzierte Unternehmen erfolgreich und findet einen Käufer. Rund jedes Fünfte schafft den Börsengang.

Bei der hohen Erfolgsquote von $51 \%$ ist allerdings zu beachten, dass die Studie kleine Wagniskapitalgeber, die weniger als elf Unternehmen finanzieren und meist geringere Erfolgschancen haben, nicht berücksichtigt.

Wie wichtig ist der erzielte Erfolg für die künftigen Geschäfte? Über alle Risikokapitalgeber hinweg zeigt die empirische Evidenz ein hohes Maß an Erfolgsbeständigkeit. Schafft es ein Risikokapitalgeber, ein weiteres von zehn finanzierten Unternehmen an die Börse bringen, erhöht sich die Wahrschein- 
lichkeit für einen Börsengang bei allen nachfolgenden Investitionen um acht Prozent. Für das nächstbeste Erfolgsszenario, einem direkten Verkauf an andere Investoren, schätzen die Forscher, dass bei zehn finanzierten Unternehmen ein zusätzlicher Verkauf die Erfolgswahrscheinlichkeit für die nachfolgenden Investitionen um vier Prozent steigert.

Mit einem zusätzlichen Börsengang unter den ersten zehn Investitionen kann ein Wagniskapitalfonds die Wahrscheinlichkeit für weitere Börsengänge unter allen seinen nachfolgenden Investitionen um 8 Prozent steigern.

Könnte der Erfolg nicht auch von anderen Einflussfaktoren als dem eigenen Erfolgsausweis abhängen? Um dafür $\mathrm{zu}$ kontrollieren, berücksichtigen die Forscher in ihren Schätzungen Faktoren wie Investitionsjahr, Bundesstaat, Branche und Investitionsphase. Damit können sie ausschliessen, dass ihre Ergebnisse von anderen Faktoren abhängen. Beispielsweise könnten erfolgreiche Wagniskapitalgeber überproportional in besonders stark wachsenden Branchen investieren, wo der Unternehmenserfolg quasi ein 'Selbstläufer' ist. Selbst unter Berücksichtigung dieser Faktoren besteht weiterhin ein starker Zusammenhang zwischen dem Anteil der Börsengänge oder der erfolgreichen Verkäufe der ersten zehn Investitionen und dem Erfolg bei den nachfolgenden Investitionen. Allerdings halbiert sich der zuvor geschätzte achtprozentige Anstieg der Wahrscheinlichkeit für einen Börsengang auf vier Prozent.

Wie lange hält eine positive Wirkung des Anfangserfolgs an? Der Effekt wird mit der Zeit immer schwächer. Mehr Erfolg bei den ersten zehn Investitionen wirkt von der elften bis zur sechzigsten nachfolgenden Investition nach. Tatsächlich nähern sich die Erfolgswahrscheinlichkeiten bei steigender Investitionsanzahl den durchschnittlichen Erfolgsraten an.

Lernen durch Erfahrung spielt für jeden Wagniskapitalgeber eine grosse Rolle. Anfänglicher Erfolg wird jedoch im Laufe der Zeit unwichtiger. Aber erst nach 60 weiteren Investitionen ist er nicht mehr relevant.

Die Bedeutung des Anfangserfolgs ist also besonders für kleine Wagniskapitalgesellschaften wichtig, die erst wenige Investitionen getätigt haben. Die grossen Gesellschaften mit einer Vielzahl getätigter Investitionen haben offensichtlich schon , ausgelernt' und können nicht mehr viel an Erfahrung dazugewinnen. Mit zunehmender Anzahl von Investitionen sollte sich also die Erfolgsquote dem Branchendurchschnitt annähern. Abb. 1 illustriert diesen Zusammenhang. Jeder Punkt repräsentiert dabei die gesamte Erfolgshistorie eines Risikokapitalgebers, also die Anzahl der getätigten Investitionen und der Anteil der Börsengänge. Im 


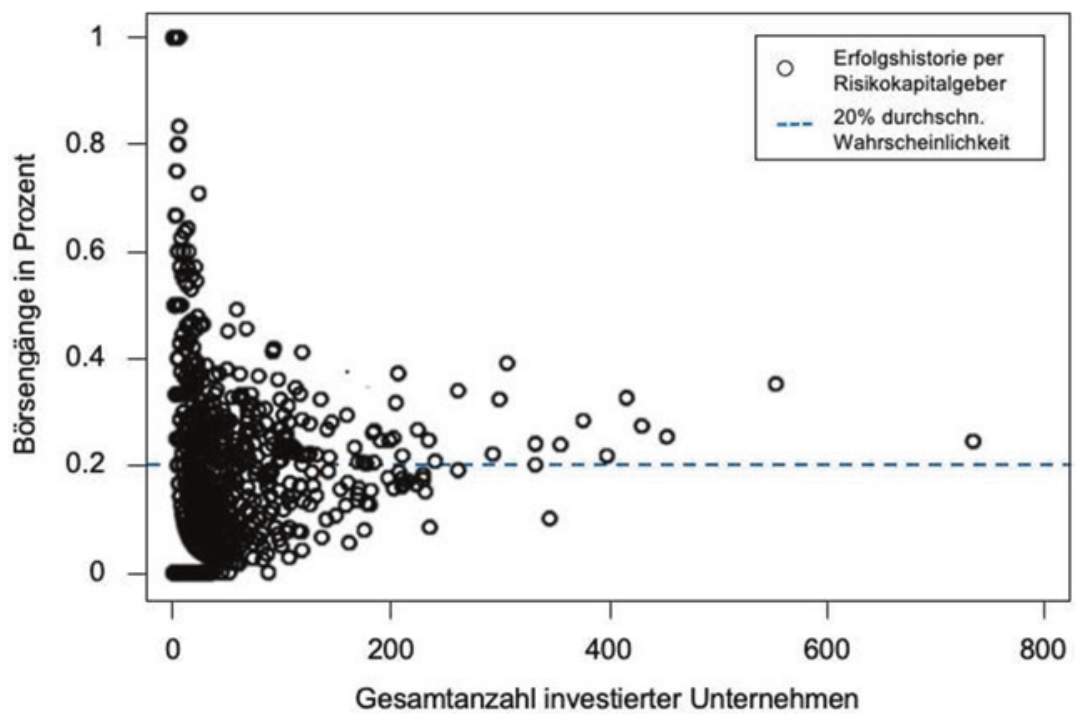

Abb. 1 Erfahrung und Performance von Risikokapitalgebern. (Quelle: Nanda u. a., 2020, Fig. 1)

Durchschnitt gelangen etwa $20 \%$ der wagnisfinanzierten Unternehmen an die Börse. Tatsächlich schwankt die Erfolgsquote der grossen Fonds mit mehr als 200 Investitionen nur wenig um diesen Durchschnittswert. Bei kleinen Wagnisgesellschaften mit weniger als 200 Investitionen streut die Erfolgsquote dagegen sehr viel stärker. Die empirischen Ergebnisse zeigen, dass in dieser Gruppe die Bedeutung des Anfangserfolgs sehr gross ist und die Erfolgsquoten der Gesellschaften stark streuen.

Worauf ist der unterschiedliche Erfolg zurückzuführen? Wagniskapitalgeber könnten sich beispielsweise in ihren Fähigkeiten unterscheiden, Startups mit hohen Erfolgsaussichten zu entdecken, diese gut zu überwachen und die Gründungsteams mit strategischer Beratung im Hinblick auf höhere Wertsteigerung zu unterstützen. Weiter könnten besonders renommierte Wagnisfinanziers bevorzugten Zugang zu den besten Neugründungen mit dem grössten Wachstumspotenzial haben, was ihre Investitionen besonders rentabel macht.

Der anfängliche Erfolg einer Wagniskapitalgesellschaft hängt fast ausschliesslich von der Investition zur richtigen Zeit am richtigen Ort ab.

Wären einige Risikokapitalgeber schlichtweg besser darin, vielversprechende Unternehmen auszuwählen oder sie zum Erfolg zu führen, sollten sie unabhängig von der vergangenen Erfolgsgeschichte höhere Erfolgsraten erzielen als ihre 
Konkurrenten, die in ähnliche Branchen investieren. Die Schätzungen zeigen jedoch, dass dies nicht der Fall ist. Ein Risikokapitalgeber, der seine ersten zehn Investitionen in jenen Marktsegmenten tätigte, die besonders hohe Chancen auf einen Börsengang versprachen, weist eine um vier Prozent höhere Chance auf einen Börsengang bei seinen nachfolgenden Investitionen auf. Andere Einflussfaktoren finden die Forscher nicht. Diese Erkenntnisse widersprechen der Ansicht, dass manche Risikokapitalgeber von Natur aus besser geeignet wären, bestimmte Jungunternehmen auszuwählen und ihren Erfolg besser zu fördern. Vielmehr scheint der Erfolg von der Investition zur richtigen Zeit am richtigen Ort abzuhängen.

Der anfängliche Erfolg der Wagniskapitalgeber hat nachhaltige Wirkung. Ein bevorzugter Zugang zu Deals in der Anfangsphase kann danach zwischen 57 und 74 Prozent der längerfristigen Erfolgsbeständigkeit erklären.

Anfangserfolg verschafft Wagniskapitalgebern Zugang zu besseren Investitionsmöglichkeiten in späteren Finanzierungsrunden und zu größeren Konsortien, welche bessere Erfolgsaussichten aufweisen. Typischerweise investiert weniger als einer von acht Risikokapitalgebern allein in ein Start-up. Jeder weitere Erfolg in den ersten Investitionsprojekten steigert die spätere Erfolgswahrscheinlichkeit um sieben bis acht Prozent. Anfänglich erfolgreiche Wagnisfinanziers ziehen grössere Investitionssummen in späteren Finanzierungsrunden an und spielen eine zentralere Rolle im Investment-Netzwerk. Anfänglicher Erfolg macht sie bei Partnern beliebt für gemeinsame Investitionen. Die Zahl der Ko-Investoren in darauffolgenden Investitionsrunden steigt.

Besserer Zugang zu besonders attraktiven Transaktionen könnte erklären, weshalb anfänglicher Erfolg über längere Zeiträume bestehen bleibt. Sowohl Startups als auch andere Investoren bevorzugen erfolgreiche Wagniskapitalgeber. Dieser Umstand vermag auch zu erklären, weshalb die Erfolgsbeständigkeit besonders für Wagniskapital charakteristisch ist und bei anderen Gesellschaften wie Investment- und Hedgefonds kaum auftritt. Reputation spielt in der Wagnisfinanzierung eine große Rolle. Unternehmen und Investoren versprechen sich von einer Zusammenarbeit mit einem besonders renommierten Wagnisfinanzier grösseren Erfolg und akzeptieren niedrigere Preise.

Zusammenfassend zeigt diese Studie, dass sich besonders bei kleineren Wagniskapitalgebern ein anfänglicher Erfolg über längere Zeit fortsetzt. Die ersten Investitionen zur richtigen Zeit am richtigen Ort erhöhen die längerfristigen Erfolgsaussichten. Diese Erfolgsbeständigkeit scheint weniger an unterschiedlichen Fähigkeiten zu liegen, sondern vielmehr am bevorzugten Zugang zu vielversprechenden Unternehmen mit besonders hohem Potenzial. 
Open Access Dieses Kapitel wird unter der Creative Commons Namensnennung 4.0 International Lizenz (http://creativecommons.org/licenses/by/4.0/deed.de) veröffentlicht, welche die Nutzung, Vervielfältigung, Bearbeitung, Verbreitung und Wiedergabe in jeglichem Medium und Format erlaubt, sofern Sie den/die ursprünglichen Autor(en) und die Quelle ordnungsgemäß nennen, einen Link zur Creative Commons Lizenz beifügen und angeben, ob Änderungen vorgenommen wurden.

Die in diesem Kapitel enthaltenen Bilder und sonstiges Drittmaterial unterliegen ebenfalls der genannten Creative Commons Lizenz, sofern sich aus der Abbildungslegende nichts anderes ergibt. Sofern das betreffende Material nicht unter der genannten Creative Commons Lizenz steht und die betreffende Handlung nicht nach gesetzlichen Vorschriften erlaubt ist, ist für die oben aufgeführten Weiterverwendungen des Materials die Einwilligung des jeweiligen Rechteinhabers einzuholen.

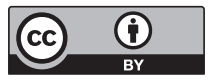

